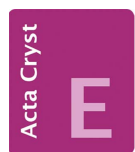

CRYSTALLOGRAPHIC COMMUNICATIONS

ISSN 2056-9890

Received 14 December 2016

Accepted 17 December 2016

Edited by $\mathrm{H}$. Stoeckli-Evans, University of Neuchâtel, Switzerland

Keywords: crystal structure; hydrazine; dinitrobenzene; di-tert-butylphenol; 3,5-di-tert-butyl4-hydroxybenzaldehyde; 2,4-dinitrophenylhydrazine; hydrogen bonding.

CCDC reference: 1523249

Supporting information: this article has supporting information at journals.iucr.org/e

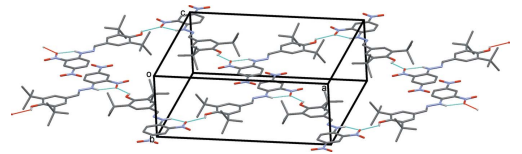

\section{Crystal structure of $(E)-2,6$-di-tert-butyl-4-\{[2-(2,4- dinitrophenyl)hydrazinylidene]methyl\}phenol}

\author{
Md. Serajul Haque Faizi, ${ }^{a}$ Necmi Dege, ${ }^{b}$ Ashanul Haque, ${ }^{a}$ Valentina A. \\ Kalibabchuk $^{\mathrm{c} *}$ and Mustafa Cemberci ${ }^{\mathrm{b}}$
}

\begin{abstract}
${ }^{a}$ Department of Chemistry, College of Science, Sultan Qaboos University, PO Box 36, Al-Khod 123, Muscat, Sultanate of , Oman, ${ }^{\mathbf{b}}$ Ondokuz Mayıs University, Arts and Sciences Faculty, Department of Physics, 55139 Samsun, Turkey, and ${ }^{c}$ Department of General Chemistry, O. O. Bohomolets National Medical University, Shevchenko Blvd. 13, 01601 Kiev, Ukraine. *Correspondence e-mail: kalibabchuk@ukr.net
\end{abstract}

The essential part (including all the non-hydrogen atoms except two methyl carbons) of the molecule of the title compound, $\mathrm{C}_{21} \mathrm{H}_{26} \mathrm{~N}_{4} \mathrm{O}_{5}$, lies on a mirror plane, which bisects the $t$-butyl groups. The conformation of the $\mathrm{C}=\mathrm{N}$ bond of this Schiff base compound is $E$, and there is an intramolecular $\mathrm{N}-\mathrm{H} \cdots \mathrm{O}$ hydrogen bond present, forming an $S(6)$ ring motif. In the crystal, molecules are linked via $\mathrm{O}-\mathrm{H} \cdots \mathrm{O}$ hydrogen bonds, forming zigzag chains propagating along the $a$-axis direction. There are no other significant intermolecular contacts present.

\section{Chemical context}

Sterically hindered phenol anti-oxidants are widely used in polymers and lubricants. They can protect polymers by increasing both their process stability and their long-term stability against oxidative degradation (Yamazaki \& Seguchi, 1997; Silin et al., 1999). Hydrazones and Schiff bases have attracted much attention for their excellent biological properties, especially for their potential pharmacological and antitumor properties (Küçükgüzel et al., 2006; Khattab, 2005; Karthikeyan et al., 2006; Okabe et al., 1993). 2,4-Dinitrophenylhydrazine is frequently used as a reagent for the characterization of aldehydes and ketones (Furniss et al., 1999). Its derivatives are widely used as dyes (Guillaumont \& Nakamura, 2000). They are also found to have versatile coordinating abilities towards different metal ions (Raj \& Kurup, 2007). The present work is a part of an ongoing structural study of Schiff bases and their utilization in the synthesis of quinoxaline derivatives (Faizi et al., 2016a), fluorescence sensors (Faizi et al., 2016b) and coordination compounds (Faizi \& Prisyazhnaya, 2015). We report herein on the synthesis and crystal structure of the title Schiff base compound with a sterically hindered phenol group.

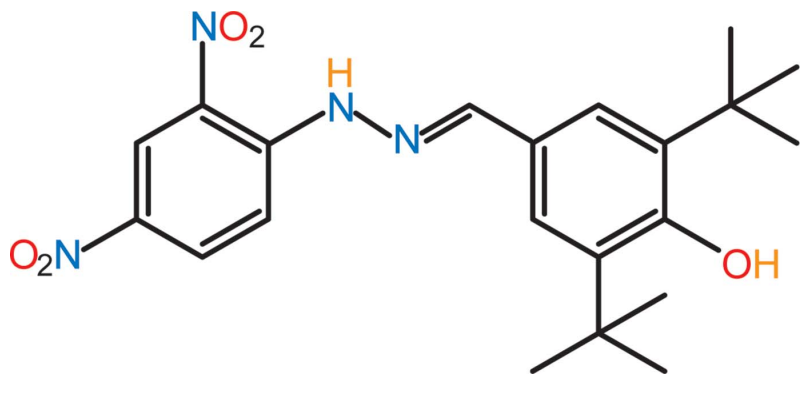




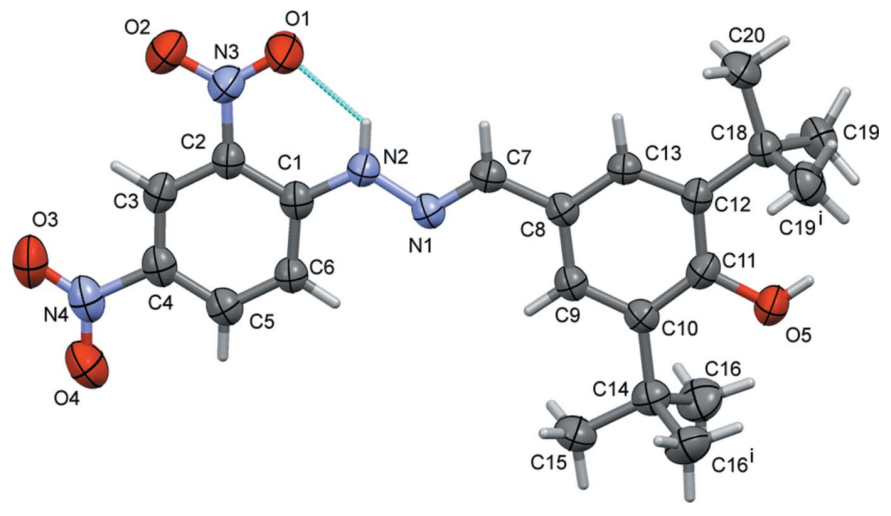

Figure 1

The molecular structure of the title compound, with atom labelling [symmetry code: (i) $x,-y+\frac{1}{2}, z$ ]. Displacement ellipsoids are drawn at the $30 \%$ probability level. The intramolecular $\mathrm{N}-\mathrm{H} \cdots \mathrm{O}$ hydrogen bond is shown as a dashed line (see Table 1)

\section{Structural commentary}

The molecular structure of the title compound is shown in Fig. 1. All the non-hydrogen atoms except C16 and C19 lie on a crystallographic mirror plane at $y=\frac{1}{4}$ : the complete tert-butyl groups are generated by mirror symmetry. The conformation of the $\mathrm{C} 7=\mathrm{N} 1$ bond of this Schiff base compound is $E$, and there is an intramolecular $\mathrm{N} 2-\mathrm{H} 2 \cdots \mathrm{O} 1$ hydrogen bond present, forming an $S(6)$ ring motif (Fig. 1 and Table 1). The $\mathrm{N} 1-\mathrm{N} 2$ bond length is $1.385(6) \AA$ and the $\mathrm{N} 1=\mathrm{C} 7$ bond length is 1.278 (7) $\AA$. The bond distances and angles in the title compound are comparable to those found in a closely related structure (Fun et al., 2013).

\section{Supramolecular features}

In the crystal, molecules are linked by $\mathrm{O}-\mathrm{H} \cdots \mathrm{O}$ hydrogen bonds, forming zigzag chains propagating along the $a$-axis direction (Fig. 2 and Table 1). There are no other significant intermolecular contacts present.
Table 1

Hydrogen-bond geometry $\left(\AA{ }^{\circ}\right)$.

\begin{tabular}{lllll}
\hline$D-\mathrm{H} \cdots A$ & $D-\mathrm{H}$ & $\mathrm{H} \cdots A$ & $D \cdots A$ & $D-\mathrm{H} \cdots A$ \\
\hline $\mathrm{N} 2-\mathrm{H} 2 \cdots \mathrm{O} 1$ & 0.86 & 1.96 & $2.583(8)$ & 129 \\
$\mathrm{O} 5-\mathrm{H} 5 O \cdots{ }^{\mathrm{i}}$ & $0.82(2)$ & $2.28(5)$ & $2.782(7)$ & $120(4)$ \\
\hline
\end{tabular}

Symmetry code: (i) $x+\frac{1}{2}, y,-z+\frac{3}{2}$.

\section{Database survey}

There are very few examples of similar compounds in the literature. To the best of our knowledge, the recent report (Bhardwaj \& Singh, 2015) of a similar compound with an hydroxy group in the ortho position, capable of visual and reversible sensing of cyanide in DMSO solution, has not been characterized crystallographically. A search of the Cambridge Structural Database (CSD, Version 5.37, update May 2016; Groom et al., 2016) revealed the structure of one very similar compound, viz. 1-(2,4-dinitrophenyl)-2-[(E)-2,4,5-trimethoxybenzylidene]hydrazine (II) (Fun et al., 2013), in which the 4-phenol group in the title compound is replaced by a trimethoxy group. In (II), the dihedral angle between the two benzene rings is $3.15(11)^{\circ}$, compared to $0^{\circ}$ in the title compound, owing to the mirror symmetry.

\section{Synthesis and crystallization}

A mixture of 3,5-di-tert-butyl-4-hydroxybenzaldehyde $0.100 \mathrm{~g}$ $(0.427 \mathrm{mmol})$ and 2,4-dinitrophenylhydrazine $(0.085 \mathrm{~g}$, $0.427 \mathrm{mmol}$ ) in methanol was refluxed for $3 \mathrm{~h}$ in the presence of a catalytic amount of glacial acetic acid. After cooling, the red-coloured precipitate was washed with hot methanol several times, and then dried, giving a red-coloured shiny crystalline compound in high yield $170 \mathrm{~g}(96 \%)$. Yellow blocklike crystals of the title compound (m.p. 372-373 K) were obtained by slow evaporation of a solution in dichloromethane and ethanol $(5: 1 v / v)$.

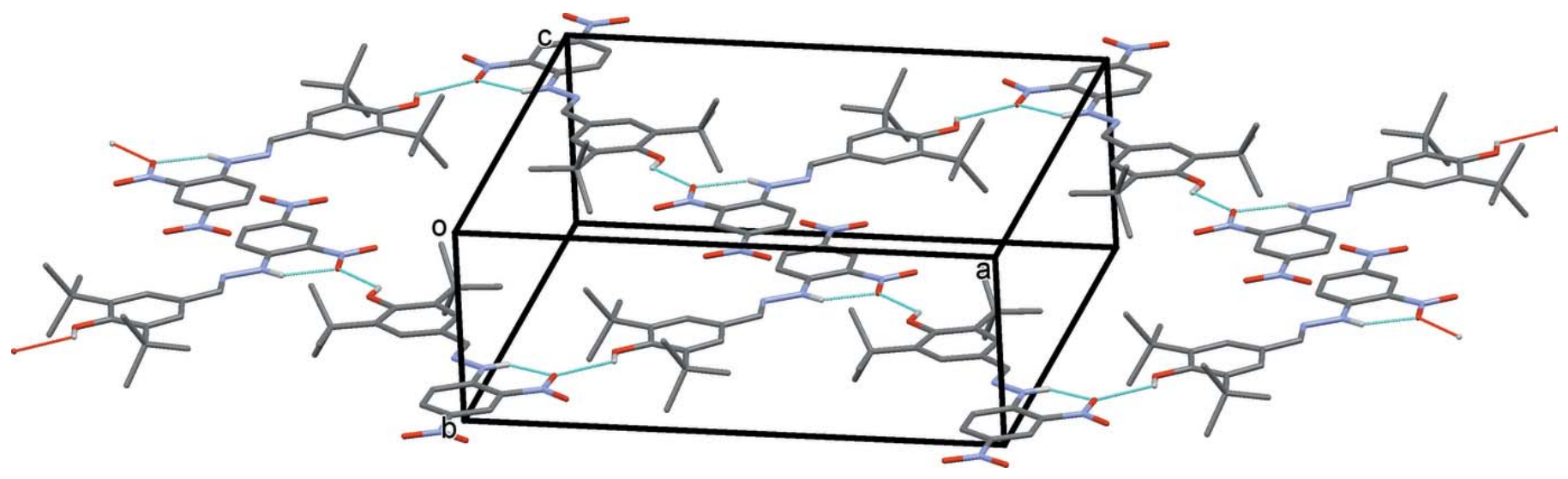

Figure 2

A view of the zigzag chains in the crystal structure of the title compound. The hydrogen bonds are shown as dashed lines (see Table 1). For clarity, only the $\mathrm{H}$ atoms involved in hydrogen bonding have been included. 
Table 2

Experimental details.

\begin{tabular}{|c|c|}
\hline \multicolumn{2}{|l|}{ Crystal data } \\
\hline Chemical formula & $\mathrm{C}_{21} \mathrm{H}_{26} \mathrm{~N}_{4} \mathrm{O}_{5}$ \\
\hline$M_{\mathrm{r}}$ & 414.46 \\
\hline Crystal system, space group & Orthorhombic, Pnma \\
\hline Temperature $(\mathrm{K})$ & 296 \\
\hline$a, b, c(\AA)$ & $18.7651(10), 6.9193(4), 17.259$ (1) \\
\hline$V\left(\AA^{3}\right)$ & $2240.9(2)$ \\
\hline$Z$ & 4 \\
\hline Radiation type & Mo $K \alpha$ \\
\hline$\mu\left(\mathrm{mm}^{-1}\right)$ & 0.09 \\
\hline Crystal size $(\mathrm{mm})$ & $0.22 \times 0.15 \times 0.11$ \\
\hline \multicolumn{2}{|l|}{ Data collection } \\
\hline Diffractometer & STOE IPDS 2 \\
\hline Absorption correction & $\begin{array}{l}\text { Integration }(X-R E D 32 ; \text { Stoe \& Cie, } \\
\text { 2002) }\end{array}$ \\
\hline$T_{\min }, T_{\max }$ & $0.982,0.994$ \\
\hline $\begin{array}{l}\text { No. of measured, independent and } \\
\text { observed }[I>2 \sigma(I)] \text { reflections }\end{array}$ & $14854,2270,912$ \\
\hline$R_{\text {int }}$ & 0.105 \\
\hline$(\sin \theta / \lambda)_{\max }\left(\AA^{-1}\right)$ & 0.606 \\
\hline \multicolumn{2}{|l|}{ Refinement } \\
\hline$R\left[F^{2}>2 \sigma\left(F^{2}\right)\right], w R\left(F^{2}\right), S$ & $0.071,0.215,0.96$ \\
\hline No. of reflections & 2270 \\
\hline No. of parameters & 178 \\
\hline No. of restraints & 2 \\
\hline H-atom treatment & $\begin{array}{l}\mathrm{H} \text { atoms treated by a mixture of } \\
\text { independent and constrained } \\
\text { refinement }\end{array}$ \\
\hline$\Delta \rho_{\max }, \Delta \rho_{\min }\left(\mathrm{e} \AA^{-3}\right)$ & $0.39,-0.16$ \\
\hline
\end{tabular}

Computer programs: $X$-AREA and X-RED32 (Stoe \& Cie, 2002), SHELXT (Sheldrick 2015a), SHELXL2016/4 (Sheldrick, 2015b), Mercury (Macrae et al., 2008), WinGX (Farrugia, 2012) and PLATON (Spek, 2009).

\section{Refinement}

Crystal data, data collection and structure refinement details are summarized in Table 2. The $\mathrm{OH} \mathrm{H}$ atom was located in a difference Fourier map and refined with a distance restraint of $0.82(2) \AA$ with $U_{\text {iso }}(\mathrm{H})=1.5 U_{\text {eq }}(\mathrm{O})$. The $\mathrm{NH}$ and C-bound $\mathrm{H}$ atoms were included in calculated positions and allowed to ride on the parent atoms: $\mathrm{N}-\mathrm{H}=0.86 \AA, \mathrm{C}-\mathrm{H}=0.93-0.96 \AA$ with $U_{\text {iso }}(\mathrm{H})=1.5 U_{\text {eq }}(\mathrm{C}-$ methyl $)$ and $1.2 U_{\text {eq }}(\mathrm{N}, \mathrm{C})$ for other $\mathrm{H}$ atoms.

\section{Acknowledgements}

The authors are grateful to Ondokuz Mayis University, Arts and Sciences Faculty, Department of Physics, Samsun, Turkey, for the X-ray data collection, and Drs Igor Fritsky and Graham Smith for helpful discussions.

\section{References}

Bhardwaj, S. \& Singh, A. K. (2015). J. Hazard. Mater. 296, 54-60.

Faizi, M. S. H., Ali, A. \& Potaskalov, V. A. (2016a). Acta Cryst. E72, 1366-1369.

Faizi, M. S. H., Gupta, S., Mohan, V. K., Jain, K. V. \& Sen, P. (2016b). Sens. Actuators B Chem. 222, 15-20.

Faizi, M. S. H. \& Prisyazhnaya, E. V. (2015). Acta Cryst. E71, m175$\mathrm{m} 176$.

Farrugia, L. J. (2012). J. Appl. Cryst. 45, 849-854.

Fun, H.-K., Chantrapromma, S., Nilwanna, B., Kobkeatthawin, T. \& Boonnak, N. (2013). Acta Cryst. E69, o1203-o1204.

Furniss, B. S., Hannaford, A. J., Smith, P. W. G. \& Tatchell, A. R. (1999). Vogel's Textbook of Practical Organic Chemistry, 5th ed. London: Longmans.

Groom, C. R., Bruno, I. J., Lightfoot, M. P. \& Ward, S. C. (2016). Acta Cryst. B72, 171-179.

Guillaumont, D. \& Nakamura, S. (2000). Dyes Pigments, 46, 85-92.

Karthikeyan, M. S., Prasad, D. J., Poojary, B., Bhat, K. S., Holla, B. S. \& Kumari, N. S. (2006). Bioorg. Med. Chem. 14, 7482-7489.

Khattab, S. N. (2005). Molecules, 10, 1218-1228.

Küçükgüzel, G., Kocatepe, A., De Clercq, E., Şahin, F. \& Güllüce, M. (2006). Eur. J. Med. Chem. 41, 353-359.

Macrae, C. F., Bruno, I. J., Chisholm, J. A., Edgington, P. R., McCabe, P., Pidcock, E., Rodriguez-Monge, L., Taylor, R., van de Streek, J. \& Wood, P. A. (2008). J. Appl. Cryst. 41, 466-470.

Okabe, N., Nakamura, T. \& Fukuda, H. (1993). Acta Cryst. C49, 16781680.

Raj, B. N. B. \& Kurup, M. R. P. (2007). Spectrochim. Acta Part A, 66, 898-903.

Sheldrick, G. M. (2015a). Acta Cryst. A71, 3-8.

Sheldrick, G. M. (2015b). Acta Cryst. C71, 3-8.

Silin, M. A., Kelaren, V. I., Abu-Ammar, V., Putkaradze, D. Kh. \& Golubeva, I. A. (1999). Pet. Chem. 40, 209-214.

Spek, A. L. (2009). Acta Cryst. D65, 148-155.

Stoe \& Cie (2002). $X$-AREA and $X$-RED32. Stoe \& Cie, Darmstadt, Germany.

Yamazaki, T. \& Seguchi, T. (1997). J. Polym. Sci. A Polym. Chem. 35, 2431-2439. 


\section{supporting information}

Acta Cryst. (2017). E73, 96-98 [https://doi.org/10.1107/S2056989016020107]

\section{Crystal structure of $(E)$-2,6-di-tert-butyl-4-\{[2-(2,4-dinitrophenyl)hydrazinyl- idene]methyl\}phenol}

Md. Serajul Haque Faizi, Necmi Dege, Ashanul Haque, Valentina A. Kalibabchuk and Mustafa Cemberci

Computing details

Data collection: $X$-AREA (Stoe \& Cie, 2002); cell refinement: $X$-AREA (Stoe \& Cie, 2002); data reduction: X-RED32

(Stoe \& Cie, 2002); program(s) used to solve structure: SHELXT (Sheldrick 2015a); program(s) used to refine structure:

SHELXL2016/4 (Sheldrick, 2015b); molecular graphics: Mercury (Macrae et al., 2008); software used to prepare material for publication: WinGX (Farrugia, 2012) and PLATON (Spek, 2009).

(E)-2,6-Di-tert-butyl-4-\{[2-(2,4-dinitrophenyl)hydrazinylidene]methyl\}phenol

Crystal data

$\mathrm{C}_{21} \mathrm{H}_{26} \mathrm{~N}_{4} \mathrm{O}_{5}$

$M_{r}=414.46$

Orthorhombic, Pnma

$a=18.7651(10) \AA$

$b=6.9193(4) \AA$

$c=17.259(1) \AA$

$V=2240.9(2) \AA^{3}$

$Z=4$

$F(000)=880$

Data collection

STOE IPDS 2

diffractometer

Radiation source: sealed X-ray tube, $12 \times 0.4$ $\mathrm{mm}$ long-fine focus

Plane graphite monochromator

Detector resolution: 6.67 pixels $\mathrm{mm}^{-1}$

rotation method scans

Absorption correction: integration

(X-RED32; Stoe \& Cie, 2002)

Refinement

Refinement on $F^{2}$

Least-squares matrix: full

$R\left[F^{2}>2 \sigma\left(F^{2}\right)\right]=0.071$

$w R\left(F^{2}\right)=0.215$

$S=0.96$

2270 reflections

178 parameters

2 restraints
$D_{\mathrm{x}}=1.228 \mathrm{Mg} \mathrm{m}^{-3}$

Mo $K \alpha$ radiation, $\lambda=0.71073 \AA$

Cell parameters from 6871 reflections

$\theta=1.1-26.2^{\circ}$

$\mu=0.09 \mathrm{~mm}^{-1}$

$T=296 \mathrm{~K}$

Block, yellow

$0.22 \times 0.15 \times 0.11 \mathrm{~mm}$

$T_{\min }=0.982, T_{\max }=0.994$

14854 measured reflections

2270 independent reflections

912 reflections with $I>2 \sigma(I)$

$R_{\text {int }}=0.105$

$\theta_{\max }=25.5^{\circ}, \theta_{\min }=1.6^{\circ}$

$h=-22 \rightarrow 22$

$k=-8 \rightarrow 8$

$l=-20 \rightarrow 20$

Secondary atom site location: difference Fourier map

Hydrogen site location: mixed

$\mathrm{H}$ atoms treated by a mixture of independent and constrained refinement

$w=1 /\left[\sigma^{2}\left(F_{0}^{2}\right)+(0.0904 P)^{2}\right]$

where $P=\left(F_{\mathrm{o}}^{2}+2 F_{\mathrm{c}}^{2}\right) / 3$

$(\Delta / \sigma)_{\max }<0.001$ 
$\Delta \rho_{\max }=0.39$ e $\AA^{-3}$

$\Delta \rho_{\min }=-0.16$ e $\AA^{-3}$

Special details

Geometry. All esds (except the esd in the dihedral angle between two 1.s. planes) are estimated using the full covariance matrix. The cell esds are taken into account individually in the estimation of esds in distances, angles and torsion angles; correlations between esds in cell parameters are only used when they are defined by crystal symmetry. An approximate (isotropic) treatment of cell esds is used for estimating esds involving l.s. planes.

Fractional atomic coordinates and isotropic or equivalent isotropic displacement parameters $\left(\hat{A}^{2}\right)$

\begin{tabular}{|c|c|c|c|c|}
\hline & $x$ & $y$ & $z$ & $U_{\text {iso }} * / U_{\text {eq }}$ \\
\hline $\mathrm{O} 1$ & $0.3313(3)$ & 0.250000 & $0.5101(4)$ & 0.172 \\
\hline $\mathrm{O} 2$ & $0.2860(3)$ & 0.250000 & $0.3982(3)$ & 0.1219 (18) \\
\hline $\mathrm{O} 3$ & $0.4249(4)$ & 0.250000 & 0.1717 (3) & 0.1208 (19) \\
\hline $\mathrm{O} 4$ & $0.5398(4)$ & 0.250000 & 0.1781 (3) & $0.134(2)$ \\
\hline O5 & $0.7461(2)$ & 0.250000 & $0.8580(3)$ & $0.0968(15)$ \\
\hline $\mathrm{H} 5 \mathrm{O}$ & $0.738(3)$ & 0.250000 & $0.9045(15)$ & $0.145^{*}$ \\
\hline N1 & $0.5301(3)$ & 0.250000 & $0.5745(3)$ & $0.0688(13)$ \\
\hline $\mathrm{N} 2$ & $0.4674(3)$ & 0.250000 & 0.5319 (3) & 0.0755 (14) \\
\hline $\mathrm{H} 2$ & 0.426908 & 0.250000 & 0.555180 & $0.091^{*}$ \\
\hline N3 & $0.3381(3)$ & 0.250000 & 0.4404 (4) & 0.0986 (19) \\
\hline N4 & $0.4801(5)$ & 0.250000 & 0.2079 (4) & $0.0960(18)$ \\
\hline $\mathrm{C} 1$ & $0.4705(4)$ & 0.250000 & $0.4537(3)$ & $0.0683(16)$ \\
\hline $\mathrm{C} 2$ & $0.4077(3)$ & 0.250000 & 0.4074 (4) & $0.0738(17)$ \\
\hline $\mathrm{C} 3$ & $0.4119(4)$ & 0.250000 & $0.3273(4)$ & $0.0743(17)$ \\
\hline H3 & 0.370779 & 0.250000 & 0.297265 & $0.089^{*}$ \\
\hline $\mathrm{C} 4$ & $0.4780(4)$ & 0.250000 & 0.2928 (4) & $0.0776(17)$ \\
\hline C5 & $0.5396(4)$ & 0.250000 & $0.3361(4)$ & 0.0778 (18) \\
\hline $\mathrm{H} 5 \mathrm{~A}$ & 0.583662 & 0.250000 & 0.311488 & $0.093 *$ \\
\hline C6 & $0.5360(3)$ & 0.250000 & $0.4143(3)$ & $0.0707(16)$ \\
\hline H6 & 0.578077 & 0.250000 & 0.442879 & $0.085^{*}$ \\
\hline $\mathrm{C} 7$ & $0.5207(3)$ & 0.250000 & 0.6478 (4) & $0.0686(16)$ \\
\hline H7 & 0.474375 & 0.250000 & 0.666931 & $0.082 *$ \\
\hline $\mathrm{C} 8$ & $0.5798(3)$ & 0.250000 & $0.7027(3)$ & $0.0642(15)$ \\
\hline C9 & $0.6498(3)$ & 0.250000 & $0.6776(3)$ & $0.0677(15)$ \\
\hline H9 & 0.659076 & 0.250000 & 0.624701 & $0.081^{*}$ \\
\hline $\mathrm{C} 10$ & $0.7066(3)$ & 0.250000 & $0.7293(3)$ & $0.0666(16)$ \\
\hline C11 & $0.6887(3)$ & 0.250000 & 0.8089 (4) & $0.0739(17)$ \\
\hline $\mathrm{C} 12$ & $0.6187(3)$ & 0.250000 & $0.8373(3)$ & $0.0663(15)$ \\
\hline C13 & $0.5656(3)$ & 0.250000 & $0.7812(3)$ & $0.0656(15)$ \\
\hline H13 & 0.518293 & 0.250000 & 0.797276 & $0.079 *$ \\
\hline $\mathrm{C} 14$ & $0.7841(3)$ & 0.250000 & 0.7007 (4) & $0.0775(18)$ \\
\hline C15 & $0.7881(4)$ & 0.250000 & 0.6117 (4) & $0.098(2)$ \\
\hline $\mathrm{H} 15 \mathrm{~A}$ & 0.764903 & 0.136762 & 0.592106 & $0.146^{*}$ \\
\hline H15B & 0.837191 & 0.250000 & 0.596024 & $0.146^{*}$ \\
\hline $\mathrm{C} 16$ & $0.8233(2)$ & $0.4334(8)$ & $0.7297(3)$ & $0.1114(18)$ \\
\hline H16A & 0.797329 & 0.546218 & 0.713820 & $0.167^{*}$ \\
\hline H16B & 0.870376 & 0.437202 & 0.708115 & $0.167^{*}$ \\
\hline $\mathrm{H} 16 \mathrm{C}$ & 0.826331 & 0.430459 & 0.785250 & $0.167 *$ \\
\hline
\end{tabular}




$\begin{array}{lllll}\text { C18 } & 0.6013(3) & 0.250000 & 0.9242(3) & 0.0755(18) \\ \text { C19 } & 0.6312(3) & 0.4323(7) & 0.9640(2) & 0.0973(16) \\ \text { H19A } & 0.679150 & 0.408097 & 0.980849 & 0.146^{*} \\ \text { H19B } & 0.602137 & 0.464417 & 1.007856 & 0.146^{*} \\ \text { H19C } & 0.631039 & 0.537943 & 0.927893 & 0.146^{*} \\ \text { C20 } & 0.5207(4) & 0.250000 & 0.9375(4) & 0.102(2) \\ \text { H20A } & 0.500334 & 0.363387 & 0.914288 & 0.154^{*} \\ \text { H20B } & 0.511112 & 0.250000 & 0.991999 & 0.154^{*}\end{array}$

Atomic displacement parameters $\left(\AA^{2}\right)$

\begin{tabular}{lllllll}
\hline & $U^{11}$ & $U^{22}$ & $U^{33}$ & $U^{12}$ & $U^{13}$ & $U^{23}$ \\
\hline O1 & $0.081(4)$ & $0.362(10)$ & $0.073(4)$ & 0.000 & $0.007(3)$ & 0.000 \\
O2 & $0.083(4)$ & $0.164(5)$ & $0.118(4)$ & 0.000 & $-0.024(3)$ & 0.000 \\
O3 & $0.147(5)$ & $0.135(5)$ & $0.080(4)$ & 0.000 & $-0.034(4)$ & 0.000 \\
O4 & $0.147(5)$ & $0.177(6)$ & $0.078(4)$ & 0.000 & $0.013(4)$ & 0.000 \\
O5 & $0.077(3)$ & $0.136(4)$ & $0.078(3)$ & 0.000 & $-0.017(3)$ & 0.000 \\
N1 & $0.072(3)$ & $0.078(3)$ & $0.056(3)$ & 0.000 & $0.000(3)$ & 0.000 \\
N2 & $0.063(3)$ & $0.103(4)$ & $0.061(3)$ & 0.000 & $-0.005(3)$ & 0.000 \\
N3 & $0.068(4)$ & $0.145(6)$ & $0.083(4)$ & 0.000 & $-0.009(4)$ & 0.000 \\
N4 & $0.130(6)$ & $0.091(4)$ & $0.068(4)$ & 0.000 & $-0.009(4)$ & 0.000 \\
C1 & $0.083(4)$ & $0.059(4)$ & $0.064(4)$ & 0.000 & $-0.008(4)$ & 0.000 \\
C2 & $0.078(5)$ & $0.081(4)$ & $0.063(4)$ & 0.000 & $-0.010(4)$ & 0.000 \\
C3 & $0.082(5)$ & $0.063(4)$ & $0.078(5)$ & 0.000 & $-0.022(4)$ & 0.000 \\
C4 & $0.102(5)$ & $0.066(4)$ & $0.065(4)$ & 0.000 & $-0.007(4)$ & 0.000 \\
C5 & $0.086(5)$ & $0.077(4)$ & $0.070(4)$ & 0.000 & $0.001(4)$ & 0.000 \\
C6 & $0.072(4)$ & $0.079(4)$ & $0.061(4)$ & 0.000 & $-0.004(3)$ & 0.000 \\
C7 & $0.067(4)$ & $0.069(4)$ & $0.069(4)$ & 0.000 & $0.003(4)$ & 0.000 \\
C8 & $0.069(4)$ & $0.066(4)$ & $0.058(4)$ & 0.000 & $0.001(3)$ & 0.000 \\
C9 & $0.077(4)$ & $0.068(4)$ & $0.058(3)$ & 0.000 & $-0.001(4)$ & 0.000 \\
C10 & $0.069(4)$ & $0.068(4)$ & $0.063(4)$ & 0.000 & $0.003(3)$ & 0.000 \\
C11 & $0.064(4)$ & $0.080(4)$ & $0.078(4)$ & 0.000 & $-0.016(4)$ & 0.000 \\
C12 & $0.069(4)$ & $0.070(4)$ & $0.060(4)$ & 0.000 & $-0.011(3)$ & 0.000 \\
C13 & $0.061(4)$ & $0.072(4)$ & $0.064(4)$ & 0.000 & $0.002(3)$ & 0.000 \\
C14 & $0.075(4)$ & $0.076(4)$ & $0.082(4)$ & 0.000 & $0.001(4)$ & 0.000 \\
C15 & $0.084(5)$ & $0.125(6)$ & $0.084(5)$ & 0.000 & $0.019(4)$ & 0.000 \\
C16 & $0.086(3)$ & $0.122(4)$ & $0.126(5)$ & $-0.028(3)$ & $0.000(3)$ & $0.000(4)$ \\
C18 & $0.076(4)$ & $0.090(5)$ & $0.061(4)$ & 0.000 & $-0.004(3)$ & 0.000 \\
C19 & $0.115(4)$ & $0.109(4)$ & $0.068(3)$ & $-0.002(3)$ & $-0.002(3)$ & $-0.013(3)$ \\
C20 & $0.089(5)$ & $0.147(7)$ & $0.071(4)$ & 0.000 & $0.017(4)$ & 0.000 \\
& & & & & &
\end{tabular}

Geometric parameters $\left(\AA,{ }^{\circ}\right)$

\begin{tabular}{llll}
\hline $\mathrm{O} 1-\mathrm{N} 3$ & $1.209(7)$ & $\mathrm{C} 9-\mathrm{H} 9$ & 0.9300 \\
$\mathrm{O} 2-\mathrm{N} 3$ & $1.219(7)$ & $\mathrm{C} 10-\mathrm{C} 11$ & $1.415(8)$ \\
$\mathrm{O} 3-\mathrm{N} 4$ & $1.210(7)$ & $\mathrm{C} 10-\mathrm{C} 14$ & $1.536(8)$ \\
$\mathrm{O} 4-\mathrm{N} 4$ & $1.232(8)$ & $\mathrm{C} 11-\mathrm{C} 12$ & $1.401(8)$ \\
$\mathrm{O} 5-\mathrm{C} 11$ & $1.370(7)$ & $\mathrm{C} 12-\mathrm{C} 13$ & $1.390(8)$
\end{tabular}




\begin{tabular}{|c|c|c|c|}
\hline $\mathrm{O} 5-\mathrm{H} 5 \mathrm{O}$ & $0.816(19)$ & $\mathrm{C} 12-\mathrm{C} 18$ & $1.536(8)$ \\
\hline $\mathrm{N} 1-\mathrm{C} 7$ & $1.278(7)$ & $\mathrm{C} 13-\mathrm{H} 13$ & 0.9300 \\
\hline $\mathrm{N} 1-\mathrm{N} 2$ & $1.385(6)$ & $\mathrm{C} 14-\mathrm{C} 15$ & $1.538(9)$ \\
\hline $\mathrm{N} 2-\mathrm{C} 1$ & $1.352(7)$ & $\mathrm{C} 14-\mathrm{C} 16$ & $1.549(6)$ \\
\hline $\mathrm{N} 2-\mathrm{H} 2$ & 0.8600 & $\mathrm{C} 14-\mathrm{C} 16^{\mathrm{i}}$ & $1.549(6)$ \\
\hline $\mathrm{N} 3-\mathrm{C} 2$ & $1.424(8)$ & C15-H15A & 0.96 \\
\hline $\mathrm{N} 4-\mathrm{C} 4$ & $1.466(8)$ & C15-H15B & 0.96 \\
\hline $\mathrm{C} 1-\mathrm{C} 6$ & $1.405(8)$ & $\mathrm{C} 15-\mathrm{H} 15 \mathrm{~A}^{\mathrm{i}}$ & 0.96 \\
\hline $\mathrm{C} 1-\mathrm{C} 2$ & $1.422(8)$ & $\mathrm{C} 16-\mathrm{H} 16 \mathrm{~A}$ & 0.9600 \\
\hline $\mathrm{C} 2-\mathrm{C} 3$ & $1.385(8)$ & C16-H16B & 0.9600 \\
\hline $\mathrm{C} 3-\mathrm{C} 4$ & $1.374(8)$ & $\mathrm{C} 16-\mathrm{H} 16 \mathrm{C}$ & 0.9600 \\
\hline $\mathrm{C} 3-\mathrm{H} 3$ & 0.9300 & $\mathrm{C} 18-\mathrm{C} 20$ & $1.530(9)$ \\
\hline $\mathrm{C} 4-\mathrm{C} 5$ & $1.376(9)$ & $\mathrm{C} 18-\mathrm{C} 19$ & $1.542(5)$ \\
\hline $\mathrm{C} 5-\mathrm{C} 6$ & $1.352(8)$ & $\mathrm{C} 18-\mathrm{C} 19^{\mathrm{i}}$ & $1.542(5)$ \\
\hline $\mathrm{C} 5-\mathrm{H} 5 \mathrm{~A}$ & 0.9300 & C19-H19A & 0.9600 \\
\hline C6- 66 & 0.9300 & C19-H19B & 0.9600 \\
\hline $\mathrm{C} 7-\mathrm{C} 8$ & $1.458(8)$ & $\mathrm{C} 19-\mathrm{H} 19 \mathrm{C}$ & 0.9600 \\
\hline $\mathrm{C} 7-\mathrm{H} 7$ & 0.9300 & $\mathrm{C} 20-\mathrm{H} 20 \mathrm{~A}$ & 0.96 \\
\hline $\mathrm{C} 8-\mathrm{C} 13$ & $1.380(7)$ & $\mathrm{C} 20-\mathrm{H} 20 \mathrm{~B}$ & 0.96 \\
\hline $\mathrm{C} 8-\mathrm{C} 9$ & $1.383(8)$ & $\mathrm{C} 20-\mathrm{H} 20 \mathrm{~A}^{\mathrm{i}}$ & 0.96 \\
\hline $\mathrm{C} 9-\mathrm{C} 10$ & $1.390(8)$ & & \\
\hline $\mathrm{C} 11-\mathrm{O} 5-\mathrm{H} 5 \mathrm{O}$ & $118(4)$ & $\mathrm{C} 12-\mathrm{C} 11-\mathrm{C} 10$ & $124.2(6)$ \\
\hline $\mathrm{C} 7-\mathrm{N} 1-\mathrm{N} 2$ & $114.1(5)$ & $\mathrm{C} 13-\mathrm{C} 12-\mathrm{C} 11$ & $115.4(5)$ \\
\hline $\mathrm{C} 1-\mathrm{N} 2-\mathrm{N} 1$ & $119.6(5)$ & $\mathrm{C} 13-\mathrm{C} 12-\mathrm{C} 18$ & $121.9(5)$ \\
\hline $\mathrm{C} 1-\mathrm{N} 2-\mathrm{H} 2$ & 120.2 & $\mathrm{C} 11-\mathrm{C} 12-\mathrm{C} 18$ & $122.7(5)$ \\
\hline $\mathrm{N} 1-\mathrm{N} 2-\mathrm{H} 2$ & 120.2 & $\mathrm{C} 8-\mathrm{C} 13-\mathrm{C} 12$ & $123.0(6)$ \\
\hline $\mathrm{O} 1-\mathrm{N} 3-\mathrm{O} 2$ & $120.6(6)$ & $\mathrm{C} 8-\mathrm{C} 13-\mathrm{H} 13$ & 118.5 \\
\hline $\mathrm{O} 1-\mathrm{N} 3-\mathrm{C} 2$ & $119.7(6)$ & $\mathrm{C} 12-\mathrm{C} 13-\mathrm{H} 13$ & 118.5 \\
\hline $\mathrm{O} 2-\mathrm{N} 3-\mathrm{C} 2$ & $119.7(6)$ & $\mathrm{C} 10-\mathrm{C} 14-\mathrm{C} 15$ & $111.5(5)$ \\
\hline $\mathrm{O} 3-\mathrm{N} 4-\mathrm{O} 4$ & $124.2(7)$ & $\mathrm{C} 10-\mathrm{C} 14-\mathrm{C} 16$ & $110.2(3)$ \\
\hline $\mathrm{O} 3-\mathrm{N} 4-\mathrm{C} 4$ & $119.5(8)$ & $\mathrm{C} 15-\mathrm{C} 14-\mathrm{C} 16$ & $107.4(4)$ \\
\hline $\mathrm{O} 4-\mathrm{N} 4-\mathrm{C} 4$ & $116.3(7)$ & $\mathrm{C} 10-\mathrm{C} 14-\mathrm{C} 16^{\mathrm{i}}$ & $110.2(3)$ \\
\hline $\mathrm{N} 2-\mathrm{C} 1-\mathrm{C} 6$ & $121.3(6)$ & $\mathrm{C} 15-\mathrm{C} 14-\mathrm{C} 16^{\mathrm{i}}$ & $107.4(4)$ \\
\hline $\mathrm{N} 2-\mathrm{C} 1-\mathrm{C} 2$ & $121.8(6)$ & $\mathrm{C} 16-\mathrm{C} 14-\mathrm{C} 16^{\mathrm{i}}$ & $110.0(6)$ \\
\hline $\mathrm{C} 6-\mathrm{C} 1-\mathrm{C} 2$ & $117.0(5)$ & $\mathrm{C} 14-\mathrm{C} 15-\mathrm{H} 15 \mathrm{~A}$ & 109.3 \\
\hline $\mathrm{C} 3-\mathrm{C} 2-\mathrm{C} 1$ & $120.9(6)$ & $\mathrm{C} 14-\mathrm{C} 15-\mathrm{H} 15 \mathrm{~B}$ & 109.2 \\
\hline $\mathrm{C} 3-\mathrm{C} 2-\mathrm{N} 3$ & $116.9(6)$ & $\mathrm{H} 15 \mathrm{~A}-\mathrm{C} 15-\mathrm{H} 15 \mathrm{~B}$ & 109.6 \\
\hline $\mathrm{C} 1-\mathrm{C} 2-\mathrm{N} 3$ & $122.3(6)$ & $\mathrm{C} 14-\mathrm{C} 15-\mathrm{H} 15 \mathrm{~A}^{\mathrm{i}}$ & $109.3(4)$ \\
\hline $\mathrm{C} 4-\mathrm{C} 3-\mathrm{C} 2$ & $118.9(6)$ & $\mathrm{H} 15 \mathrm{~A}-\mathrm{C} 15-\mathrm{H} 15 \mathrm{~A}^{\mathrm{i}}$ & 109.7 \\
\hline $\mathrm{C} 4-\mathrm{C} 3-\mathrm{H} 3$ & 120.5 & $\mathrm{H} 15 \mathrm{~B}-\mathrm{C} 15-\mathrm{H} 15 \mathrm{~A}^{\mathrm{i}}$ & 109.6 \\
\hline $\mathrm{C} 2-\mathrm{C} 3-\mathrm{H} 3$ & 120.5 & $\mathrm{C} 14-\mathrm{C} 16-\mathrm{H} 16 \mathrm{~A}$ & 109.5 \\
\hline $\mathrm{C} 3-\mathrm{C} 4-\mathrm{C} 5$ & $121.5(6)$ & $\mathrm{C} 14-\mathrm{C} 16-\mathrm{H} 16 \mathrm{~B}$ & 109.5 \\
\hline $\mathrm{C} 3-\mathrm{C} 4-\mathrm{N} 4$ & $117.2(7)$ & $\mathrm{H} 16 \mathrm{~A}-\mathrm{C} 16-\mathrm{H} 16 \mathrm{~B}$ & 109.5 \\
\hline $\mathrm{C} 5-\mathrm{C} 4-\mathrm{N} 4$ & $121.3(7)$ & $\mathrm{C} 14-\mathrm{C} 16-\mathrm{H} 16 \mathrm{C}$ & 109.5 \\
\hline $\mathrm{C} 6-\mathrm{C} 5-\mathrm{C} 4$ & $120.1(7)$ & $\mathrm{H} 16 \mathrm{~A}-\mathrm{C} 16-\mathrm{H} 16 \mathrm{C}$ & 109.5 \\
\hline $\mathrm{C} 6-\mathrm{C} 5-\mathrm{H} 5 \mathrm{~A}$ & 120.0 & $\mathrm{H} 16 \mathrm{~B}-\mathrm{C} 16-\mathrm{H} 16 \mathrm{C}$ & 109.5 \\
\hline $\mathrm{C} 4-\mathrm{C} 5-\mathrm{H} 5 \mathrm{~A}$ & 120.0 & $\mathrm{C} 20-\mathrm{C} 18-\mathrm{C} 12$ & $110.9(5)$ \\
\hline
\end{tabular}




$\begin{array}{ll}\mathrm{C} 5-\mathrm{C} 6-\mathrm{C} 1 & 121.7(6) \\ \mathrm{C} 5-\mathrm{C} 6-\mathrm{H} 6 & 119.2 \\ \mathrm{C} 1-\mathrm{C} 6-\mathrm{H} 6 & 119.2 \\ \mathrm{~N} 1-\mathrm{C} 7-\mathrm{C} 8 & 122.6(6) \\ \mathrm{N} 1-\mathrm{C} 7-\mathrm{H} 7 & 118.7 \\ \mathrm{C} 8-\mathrm{C} 7-\mathrm{H} 7 & 118.7 \\ \mathrm{C} 13-\mathrm{C} 8-\mathrm{C} 9 & 119.4(6) \\ \mathrm{C} 13-\mathrm{C} 8-\mathrm{C} 7 & 119.4(6) \\ \mathrm{C} 9-\mathrm{C} 8-\mathrm{C} 7 & 121.2(5) \\ \mathrm{C} 8-\mathrm{C} 9-\mathrm{C} 10 & 121.8(6) \\ \mathrm{C} 8-\mathrm{C} 9-\mathrm{H} 9 & 119.1 \\ \mathrm{C} 10-\mathrm{C} 9-\mathrm{H} 9 & 119.1 \\ \mathrm{C} 9-\mathrm{C} 10-\mathrm{C} 11 & 116.2(6) \\ \mathrm{C} 9-\mathrm{C} 10-\mathrm{C} 14 & 121.4(5) \\ \mathrm{C} 11-\mathrm{C} 10-\mathrm{C} 14 & 122.4(5) \\ \mathrm{O} 5-\mathrm{C} 11-\mathrm{C} 12 & 121.4(6) \\ \mathrm{O} 5-\mathrm{C} 11-\mathrm{C} 10 & 114.4(6) \\ \mathrm{C} 7-\mathrm{N} 1-\mathrm{N} 2-\mathrm{C} 1 & \\ \mathrm{~N} 1-\mathrm{N} 2-\mathrm{C} 1-\mathrm{C} 6 & 180.000(1) \\ \mathrm{N} 1-\mathrm{N} 2-\mathrm{C} 1-\mathrm{C} 2 & 0.000(1) \\ \mathrm{N} 2-\mathrm{C} 1-\mathrm{C} 2-\mathrm{C} 3 & 180.000(1) \\ \mathrm{C} 6-\mathrm{C} 1-\mathrm{C} 2-\mathrm{C} 3 & 180.000(1) \\ \mathrm{N} 2-\mathrm{C} 1-\mathrm{C} 2-\mathrm{N} 3 & 0.000(1) \\ \mathrm{C} 6-\mathrm{C} 1-\mathrm{C} 2-\mathrm{N} 3 & 0.000(1) \\ \mathrm{O} 1-\mathrm{N} 3-\mathrm{C} 2-\mathrm{C} 3 & 180.000(1) \\ \mathrm{O} 2-\mathrm{N} 3-\mathrm{C} 2-\mathrm{C} 3 & 180.000(1) \\ \mathrm{O} 1-\mathrm{N} 3-\mathrm{C} 2-\mathrm{C} 1 & 0.000(1) \\ \mathrm{O} 2-\mathrm{N} 3-\mathrm{C} 2-\mathrm{C} 1 & 0.000(1) \\ \mathrm{C} 1-\mathrm{C} 2-\mathrm{C} 3-\mathrm{C} 4 & 180.000(1) \\ \mathrm{N} 3-\mathrm{C} 2-\mathrm{C} 3-\mathrm{C} 4 & 0.000(1) \\ \mathrm{C} 2-\mathrm{C} 3-\mathrm{C} 4-\mathrm{C} 5 & 180.000(1) \\ \mathrm{C} 2-\mathrm{C} 3-\mathrm{C} 4-\mathrm{N} 4 & 0.000(1) \\ \mathrm{O} 3-\mathrm{N} 4-\mathrm{C} 4-\mathrm{C} 3 & 180.000(1) \\ \mathrm{O} 4-\mathrm{N} 4-\mathrm{C} 4-\mathrm{C} 3 & 0.000(1) \\ \mathrm{O} 3-\mathrm{N} 4-\mathrm{C} 4-\mathrm{C} 5 & 180.000(1) \\ \mathrm{O} 4-\mathrm{N} 4-\mathrm{C} 4-\mathrm{C} 5 & 180.000(1) \\ \mathrm{C} 3-\mathrm{C} 4-\mathrm{C} 5-\mathrm{C} 6 & 0.000(1) \\ \mathrm{N} 4-\mathrm{C} 4-\mathrm{C} 5-\mathrm{C} 6 & 0.000(1) \\ \mathrm{C} 4-\mathrm{C} 5-\mathrm{C} 6-\mathrm{C} 1 & 180.000(1) \\ \mathrm{N} 2-\mathrm{C} 1-\mathrm{C} 6-\mathrm{C} 5 & 0.000(1) \\ \mathrm{C} 2-\mathrm{C} 1-\mathrm{C} 6-\mathrm{C} 5 & 180.000(1) \\ \mathrm{N} 2-\mathrm{N} 1-\mathrm{C} 7-\mathrm{C} 8 & (1) \\ \mathrm{N} 1-\mathrm{C} 7-\mathrm{C} 7-\mathrm{C} 8-\mathrm{C} 13 & \mathrm{C} 9 \\ & \end{array}$

$\begin{array}{ll}\mathrm{C} 20-\mathrm{C} 18-\mathrm{C} 19 & 107.1(4) \\ \mathrm{C} 12-\mathrm{C} 18-\mathrm{C} 19 & 110.9(3) \\ \mathrm{C} 20-\mathrm{C} 18-\mathrm{C} 19^{\mathrm{i}} & 107.1(4) \\ \mathrm{C} 12-\mathrm{C} 18-\mathrm{C} 19^{\mathrm{i}} & 110.9(3) \\ \mathrm{C} 19-\mathrm{C} 18-\mathrm{C} 19^{\mathrm{i}} & 109.8(5) \\ \mathrm{C} 18-\mathrm{C} 19-\mathrm{H} 19 \mathrm{~A} & 109.5 \\ \mathrm{C} 18-\mathrm{C} 19-\mathrm{H} 19 \mathrm{~B} & 109.5 \\ \mathrm{H} 19 \mathrm{~A}-\mathrm{C} 19-\mathrm{H} 19 \mathrm{~B} & 109.5 \\ \mathrm{C} 18-\mathrm{C} 19-\mathrm{H} 19 \mathrm{C} & 109.5 \\ \mathrm{H} 19 \mathrm{~A}-\mathrm{C} 19-\mathrm{H} 19 \mathrm{C} & 109.5 \\ \mathrm{H} 19 \mathrm{~B}-\mathrm{C} 19-\mathrm{H} 19 \mathrm{C} & 109.5 \\ \mathrm{C} 18-\mathrm{C} 20-\mathrm{H} 20 \mathrm{~A} & 109.3 \\ \mathrm{C} 18-\mathrm{C} 20-\mathrm{H} 20 \mathrm{~B} & 109.5 \\ \mathrm{H} 20 \mathrm{~A}-\mathrm{C} 20-\mathrm{H} 20 \mathrm{~B} & 109.6 \\ \mathrm{C} 18-\mathrm{C} 20-\mathrm{H} 20 \mathrm{~A}^{\mathrm{i}} & 109.3(5) \\ \text { H20A-C20-H20A } & 109.6 \\ \mathrm{H} 20 \mathrm{~B}-\mathrm{C} 20-\mathrm{H} 20 \mathrm{~A}^{\mathrm{i}} & 109.6\end{array}$

$\mathrm{C} 7-\mathrm{C} 8-\mathrm{C} 9-\mathrm{C} 10 \quad 180.000(1)$

$\mathrm{C} 8-\mathrm{C} 9-\mathrm{C} 10-\mathrm{C} 11 \quad 0.000(2)$

$\mathrm{C} 8-\mathrm{C} 9-\mathrm{C} 10-\mathrm{C} 14 \quad 180.000(1)$

$\mathrm{C} 9-\mathrm{C} 10-\mathrm{C} 11-\mathrm{O} 5 \quad 180.000$ (1)

$\mathrm{C} 14-\mathrm{C} 10-\mathrm{C} 11-\mathrm{O} 5 \quad 0.000$ (1)

$\mathrm{C} 9-\mathrm{C} 10-\mathrm{C} 11-\mathrm{C} 12 \quad 0.000(2)$

$\mathrm{C} 14-\mathrm{C} 10-\mathrm{C} 11-\mathrm{C} 12 \quad 180.000$ (1)

$\mathrm{O} 5-\mathrm{C} 11-\mathrm{C} 12-\mathrm{C} 13 \quad 180.000(1)$

$\mathrm{C} 10-\mathrm{C} 11-\mathrm{C} 12-\mathrm{C} 13 \quad 0.000(2)$

$\mathrm{O} 5-\mathrm{C} 11-\mathrm{C} 12-\mathrm{C} 18 \quad 0.000(2)$

$\mathrm{C} 10-\mathrm{C} 11-\mathrm{C} 12-\mathrm{C} 18 \quad 180.000(2)$

$\mathrm{C} 9-\mathrm{C} 8-\mathrm{C} 13-\mathrm{C} 12 \quad 0.000(2)$

$\mathrm{C} 7-\mathrm{C} 8-\mathrm{C} 13-\mathrm{C} 12 \quad 180.000$ (1)

$\mathrm{C} 11-\mathrm{C} 12-\mathrm{C} 13-\mathrm{C} 8 \quad 0.000(2)$

$\mathrm{C} 18-\mathrm{C} 12-\mathrm{C} 13-\mathrm{C} 8 \quad 180.000(2)$

$\mathrm{C} 9-\mathrm{C} 10-\mathrm{C} 14-\mathrm{C} 15 \quad 0.000(2)$

$\mathrm{C} 11-\mathrm{C} 10-\mathrm{C} 14-\mathrm{C} 15 \quad 180.000$ (1)

$\mathrm{C} 9-\mathrm{C} 10-\mathrm{C} 14-\mathrm{C} 16 \quad 119.2$ (4)

$\mathrm{C} 11-\mathrm{C} 10-\mathrm{C} 14-\mathrm{C} 16 \quad-60.8$ (4)

$\mathrm{C} 9-\mathrm{C} 10-\mathrm{C} 14-\mathrm{C} 16^{\mathrm{i}} \quad-119.2(4)$

$\mathrm{C} 11-\mathrm{C} 10-\mathrm{C} 14-\mathrm{C}^{\mathrm{i}} \quad 60.8$ (4)

$\mathrm{C} 13-\mathrm{C} 12-\mathrm{C} 18-\mathrm{C} 20 \quad 0.000$ (2)

$\mathrm{C} 11-\mathrm{C} 12-\mathrm{C} 18-\mathrm{C} 20 \quad 180.000(2)$

$\mathrm{C} 13-\mathrm{C} 12-\mathrm{C} 18-\mathrm{C} 19 \quad-118.8$ (4)

$\mathrm{C} 11-\mathrm{C} 12-\mathrm{C} 18-\mathrm{C} 19 \quad 61.2(4)$

$\mathrm{C} 13-\mathrm{C} 12-\mathrm{C} 18-\mathrm{C} 19^{\mathrm{i}} \quad 118.8(4)$

$\mathrm{C} 11-\mathrm{C} 12-\mathrm{C} 18-\mathrm{C} 19^{\mathrm{i}} \quad-61.2(4)$

Symmetry code: (i) $x,-y+1 / 2, z$. 
Hydrogen-bond geometry $\left(\AA,{ }^{\circ}\right)$

\begin{tabular}{lllll}
\hline$D-\mathrm{H} \cdots A$ & $D-\mathrm{H}$ & $\mathrm{H} \cdots A$ & $D \cdots A$ & $D-\mathrm{H} \cdots A$ \\
\hline $\mathrm{N} 2-\mathrm{H} 2 \cdots \mathrm{O} 1$ & 0.86 & 1.96 & $2.583(8)$ & 129 \\
$\mathrm{O} 5-\mathrm{H} 5 O \cdots \mathrm{O} 1^{\mathrm{ii}}$ & $0.82(2)$ & $2.28(5)$ & $2.782(7)$ & $120(4)$ \\
\hline
\end{tabular}

Symmetry code: (ii) $x+1 / 2, y,-z+3 / 2$. 